\title{
Development of Quadruped Robot with Pneumatic Actuator
}

\author{
Masahiro Takaiwa*1 ${ }^{* 1}$ Toshiro Noritsugu*1, Zilei Zhao*1 and Daisuke Sasaki*1 \\ *1 The Graduate School of Natural Science and Technology, Okayama University. \\ 3-1-1, Kitaku Tsushimanaka, Okayama, 700-8530, Japan
}

\begin{abstract}
:
In this study, we develop a quadruped walking robot, assuming, such as, a human cooperative tasks in a construction building or a rescue activity in a disaster environment. Pneumatic cylinders are employed as driving actuators since it has explosion proof and inherent compliance. Owing to the compliance feature external force is easily estimated based on a inner pressure of cylinder. We compose a compliance control system on each leg based on the estimated floor reaction force with a disturbance observer.

After mentioning a overview of the developed quadruped walking robot, we describe a kinematics, a statics and a control system of each leg. We propose a motion control strategy based on the floor reaction force estimated using a disturbance observer. The validity of the proposed control system is verified through some experiments.
\end{abstract}

\section{INTRODUCTION}

In order to make a robot to perform several services in an outer field, its moving mechanism is important issue. The legged mechanism is indispensable especially for the rough terrain environment since it can select a contact point arbitrary. Researches for the multi-legged robot have been classified to the walking mechanism, such as ZMP based walking ${ }^{(1)}$ or CPG one ${ }^{(2)}$ and its application, such as, forestry ${ }^{(3)}$, demining ${ }^{(4)}$, steep slope operation ${ }^{(5)}$, volcano investigation $^{(6)}$ and entertainment one ${ }^{(7)}$. Viewing from the point of size, most of the developed multi-legged robot are small one except for the some huge robot.

One of the application of multi-legged robot is a task with human, such as cooperative carrying objects in a construction building or rescue victims in a disaster area. Such a robot should be the same with human from the view point of the size and the power level and also it should hold a compliance property.

We develop a quadruped robot using pneumatic actuators on the assumption of applying it to the above mentioned human friendly tasks. Employing pneumatic actuators bring some advantages as follows. As you know, compliance is necessary not only for the flexible property as human friendly robot but also for a stable gait. Therefore we compose a position based compliance control system on each leg. In this case, force/moment sensors are generally required but it is not realistic from a view of cost and reliability especially for a field robot. In a pneumatic actuator, the control variable(stroke of piston) is easily affected by the external force, which means simultaneously that the external force is easily estimated based on the pressure. We propose a compliance control system using an estimated floor reaction force with disturbance observer. The validity of the proposed control system is verified through some experiments.

\section{OVERVIEW of PNEUMATIC QUADRUPED ROBOT}

2.1 schematic diagram Fig.1 shows an overview of the developed quadruped robot. Serial-linkage legs are placed at each corner of the base plate $(1,000[\mathrm{~mm}]$ in length and 773 [mm] in width). The total weight is about 209 [kg]. The position and orientation of the base plate and the position of each leg is described with a coordinate frame $\Sigma_{b o d y}$ placed at the center of base plate and hand coordinate is defined as $h=[x, y, z]$. As shown in (a), the legs are placed being symmetry with respect to the front and the rear considering the same motion property for the both direction.

Figure (b),(c) shows a detail mechanical structure of a leg. A leg is connected to the base plate with a 2 D.O.F. universal joint(joint-1,joint-2). At joint-1, a rotational shaft is inserted and mechanically fixed and the shaft is held by a base plate with shaft bearings. Therefore a cylinder- $1(80 \mathrm{~mm}$ in diameter) drives a whole leg around joint- 1 by rotating the shaft. The head side of a cylinder $-2(80[\mathrm{~mm}]$ in diameter $)$ is also mechanically attached to a shaft with a bearing and it gives a rotational motion around joint-2 axis. A cylinder$3(100[\mathrm{~mm}]$ in diameter $)$ is mounted on the Leg1 to yields a rotation motion around joint-3. An ankle joint has also 2 D.O.F. universal joint and they are equipped with passive dampers.

Here we introduce a joint coordinate $\theta=\left[\theta_{1}, \theta_{2}, \theta_{3}\right]^{T}$ corresponding to the rotational angle around joint-1,2,3 and they are measured by rotary encoders. Figure (d) shows the moving area of forward right side leg in $y-z$ plane, where an origin point of $h$ is set at the joint-1,2 for simplicity. It can be seen that about $700 \mathrm{~mm}$ stroke for $y$ direction(forward direction) is obtained at the standard height shown by a dashed line in the figure.

Fig. 2 shows a driving circuit for one pneumatic cylinder. Pressure in both chambers are measured by pressure sensors and they are regulated by pressure control valve, 


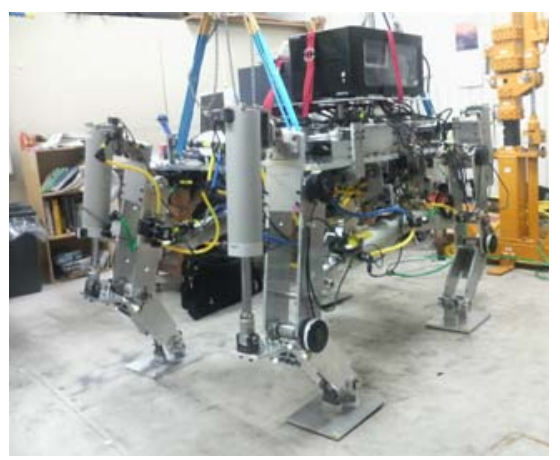

(a) Whole view

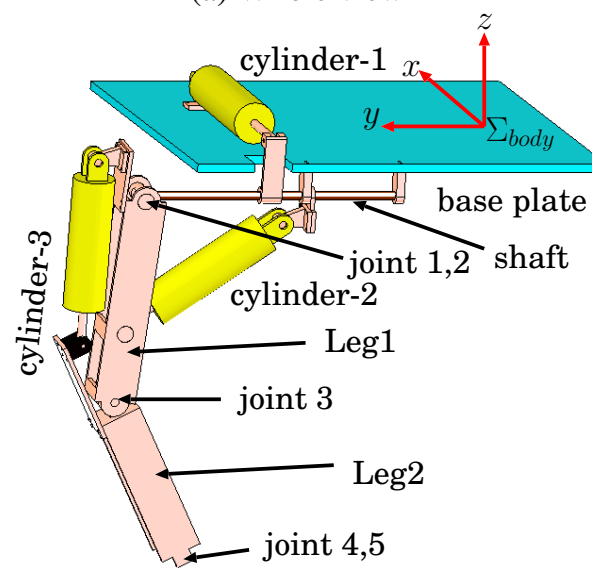

(b) schematic diagram of leg mechanism

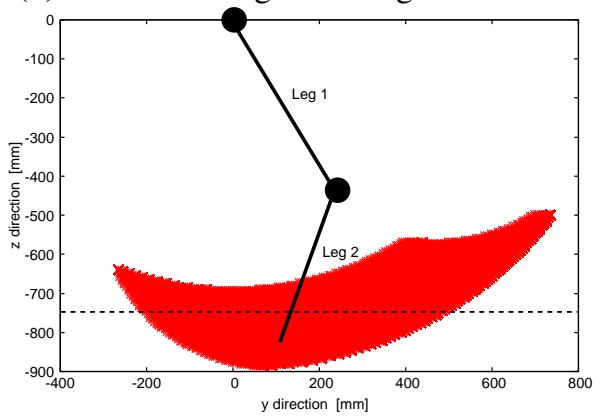

(c) moving area

Fig. 1 Outline of pneumatic quadruped robot

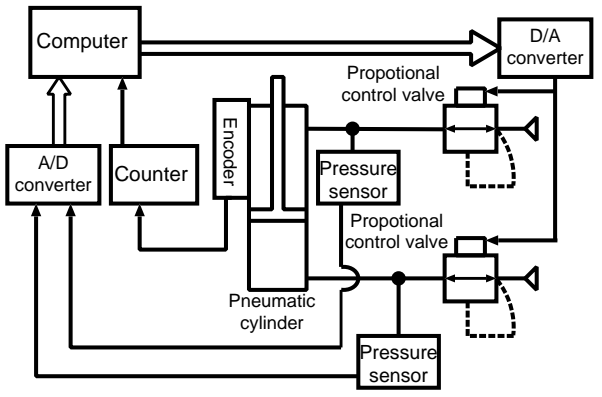

Fig. 2 Pneumatic driving circuit

respectively. These 2 control valves are controlled with one control signal to behave as a SISO system. A joint angle is detected by a rotary encoder. The control system must be

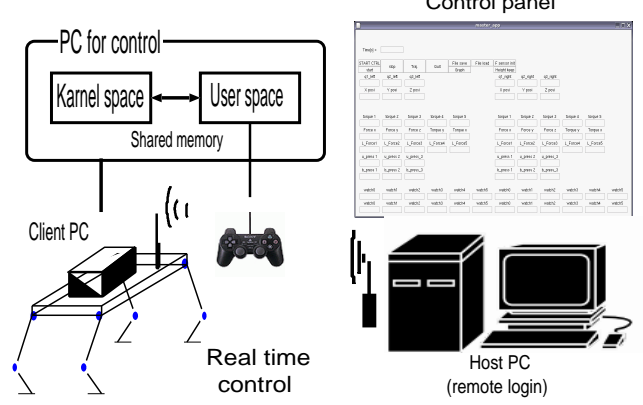

Fig. 3 Control Environment

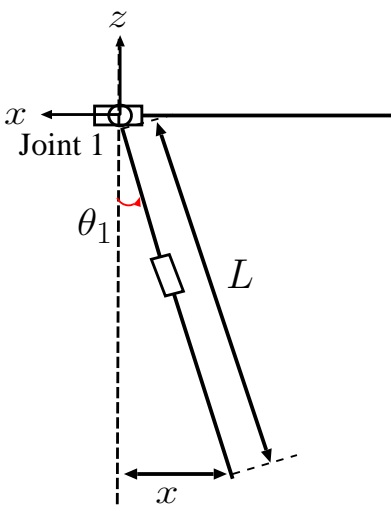

(a) Front view

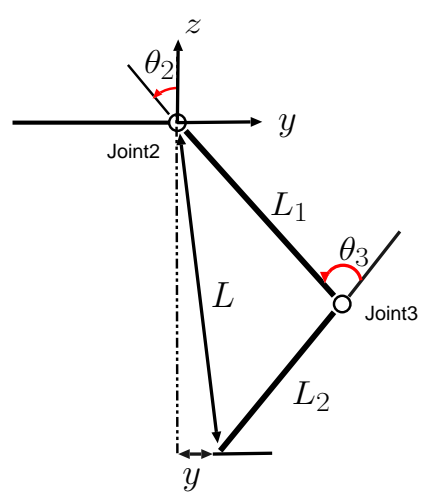

(b) Side view
Fig. 4 Schematic diagram of a leg

implemented as a real-time control system. In this study, RT-Linux, the extension of linux to the real-time system, is employed, where a sampling interval is set to be $5 \mathrm{~ms}$.

Fig. 3 shows a whole control environment. A computer execute the real-time servo system is mounted on a robot and it is connected with remote host computer via wireless Internet. An operator control log in the computer on a robot with remote $\log$ in function. In a RT-Linux, real-time control is executed in a kernel space and the control variables are displayed on a GUI screen in the user space via shared memory.

2.2 kinematics Fig.4 shows a schematic diagram of forward right side leg. Owing to the mechanical simplicity of a leg, the kinematics can be easily solved.

$$
\left\{\begin{array}{l}
\theta_{1}=\sin ^{-1} \frac{x}{L} \\
\theta_{2}=\cos ^{-1} \frac{L_{1}^{2}+L^{2}-L_{2}^{2}}{2 L_{1} L}+\sin ^{-1} \frac{y}{L} \\
\theta_{3}=\cos ^{-1}\left(-\frac{L_{1}^{2}+L_{2}^{2}-L^{2}}{2 L_{1} L_{2}}\right)
\end{array}\right.
$$

,where $L_{1}, L_{2}$ are length of leg-1 and leg-2, respectively and $L=\sqrt{x^{2}+y^{2}+z^{2}}$ is distance between a center point of universal joint(joint-1,joint-2) and a tip of leg-2.

\section{CONTROL SYSTEM}

3.1 compliance control system In generally, a position control is composed on legs for this kind of walking 
robot and mostly 1-type controller(one integrator in an open-loop control system) is employed. In this case, if a deviation between the reference position and current one is occurred under the state of contact with ground, the position controller, of course, works to eliminate the deviation by an integral effect. Consequently, an inner force affects each other among legs, which may lead the robot be unstable. One of the solutions for this problem is to give compliance to the leg. Holding a compliance property on a leg contributes to not only stabilize the robot but absorb the step in rough terrain without complex control strategy.

In order to implement a compliance control, a force/moment sensor is necessary to mount at the ankle joint of a leg, however, it is not practical from a view of a cost and maintenance for the field working robots. In a pneumatic actuator, the control variable(stroke of piston) is easily affected by the external force, which means at the same time that the external force is easily estimated based on the pressure. Therefore we propose a compliance control system using not a force/moment sensor but a estimation function of disturbance observer.

Fig.5 shows the proposed compliance control system. Figure (a) is a schematic diagram of a position based compliance control system, where $\tau$ is a estimated external torque and it is fed back as floor reaction force $F$ through a transpose of Jacobi matrix $J^{T}$ from a principle of virtual work. $K=\operatorname{diag}\left\{K_{x}, K_{y}, K_{z}\right\}$ is a stiffness matrix.

Figure (b) shows a concrete description of a position control part in figure (a)(dotted line box), where $F_{g}(s)=$ $P_{1}(s) A_{1}-P_{2}(s) A_{2}, r, J, D$ are a generated force of cylinder, moment arm, inertial moment, damping coefficient, respectively. First, a disturbance observer is applied to the mechanical transfer part $P_{s}(s)$. If the nominal model $P_{n}(s)$ is the same with $P_{k}(s)$, then the estimated disturbance $D(s)$ contains external force and external torque $\tau$ is derived as $r D(s)$. A doubled line box in the figure is generation force control system and it is concretely described in the figure (c). A disturbance observer is also applied to this control system, which works the closed loop transfer function from the reference $F_{r}$ to $F_{g}$ to match the nominal model $P_{p n}(s)$ and reduce the influence of piston velocity which works as a disturbance. The time constant of filter $Q(s), T_{q}$ plays an important role on the control performance and stability of the closed loop control system. In this study, we regulated it on trial and error.

3.2 Estimation of body orientation In order to carry out a locomotion, it is important to estimate the relative current posture of the base plate for the ground. We set a coordinate frame $\Sigma_{\text {body }}$ on the center point of the base plate as shown in Fig.6(a). In a static gait, 3 legs are always contact with ground. Now we set position vectors $h_{1}, h_{2}, h_{3}$ for the contacting 3 legs. Then a unit normal vector $n$ for the plane composed by the 3 position vectors is described as follows.

$$
n=\frac{\left(h_{2}-h_{3}\right) \times\left(h_{4}-h_{3}\right)}{\left|h_{2}-h_{3}\right|\left|h_{4}-h_{3}\right|}
$$

We define the posture of base plane with $\operatorname{roll}(\phi)-\operatorname{pitch}(\theta)$ $\operatorname{yaw}(\psi)$ notation, then the following relation is obtained.

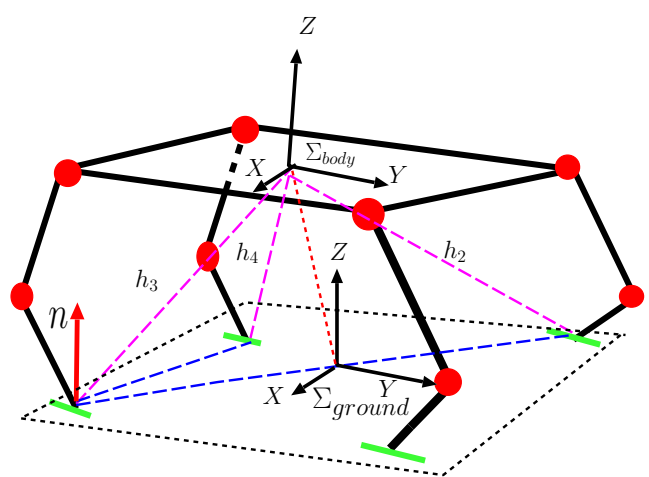

(a) orientation detection

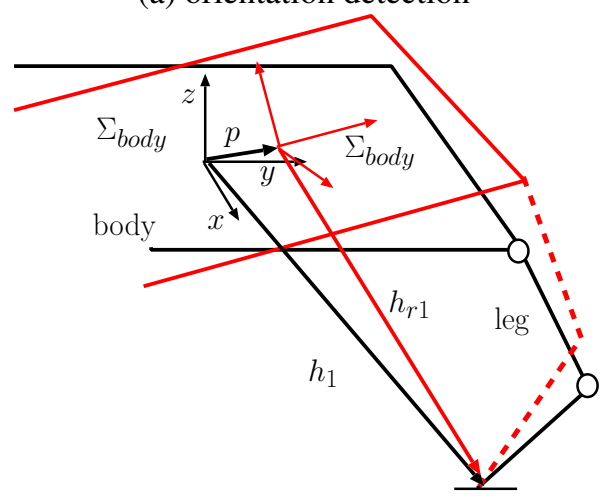

(b) orientation compensation

Fig. 6 Orientation detection and compensation

$$
R \cdot n=\left[\begin{array}{lll}
0 & 0 & 1
\end{array}\right]^{T}
$$

, where $R$ is a rotational matrix.

Eq.(3) is calculated as follows with roll angle $\phi=0$.

$$
\left\{\begin{array}{l}
\cos \theta n_{x}+\sin \theta \sin \psi n_{y}+\sin \theta \cos \psi n_{z}=0 \\
\cos \psi n_{y}-\sin \psi n_{z}=0
\end{array}\right.
$$

Solving Eq.(4), the posture angle is obtained as

$$
\left\{\begin{array}{l}
\psi=\tan ^{-1} \frac{n_{y}}{n_{z}} \\
\theta=\tan ^{-1} \frac{-n_{x}}{\sin \psi n_{y}+\cos \psi n_{z}}
\end{array}\right.
$$

Fig.6(b) shows the compensation of the position and orientation, where red line is the reference posture and black one shows the current one. The reference position vector is described as follows.

$$
h_{r}=R^{T}(h-p)
$$

,where $p$ is a deviation from reference position.

\section{EXPERIMENTAL RESULTS}

4.1 force estimation and compliance control Fig.7 shows the property of force estimation using a disturbance observer. In the state the robot is suspended by a crane as shown in the figure (a), a mass of $20 \mathrm{~kg}$ hangs from a 


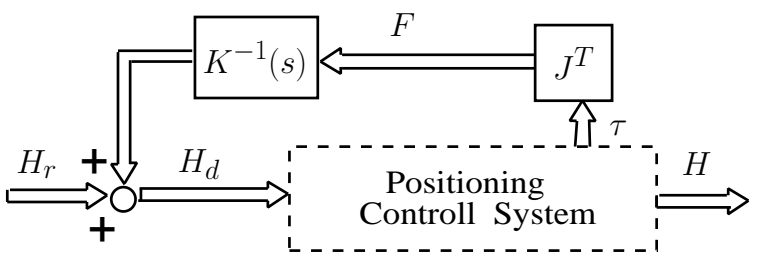

(a) Position based compliance control system

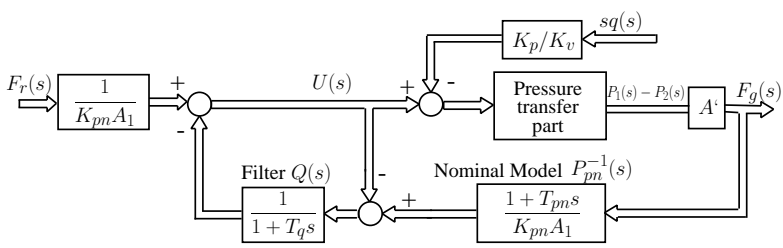

(c) Generation force control system

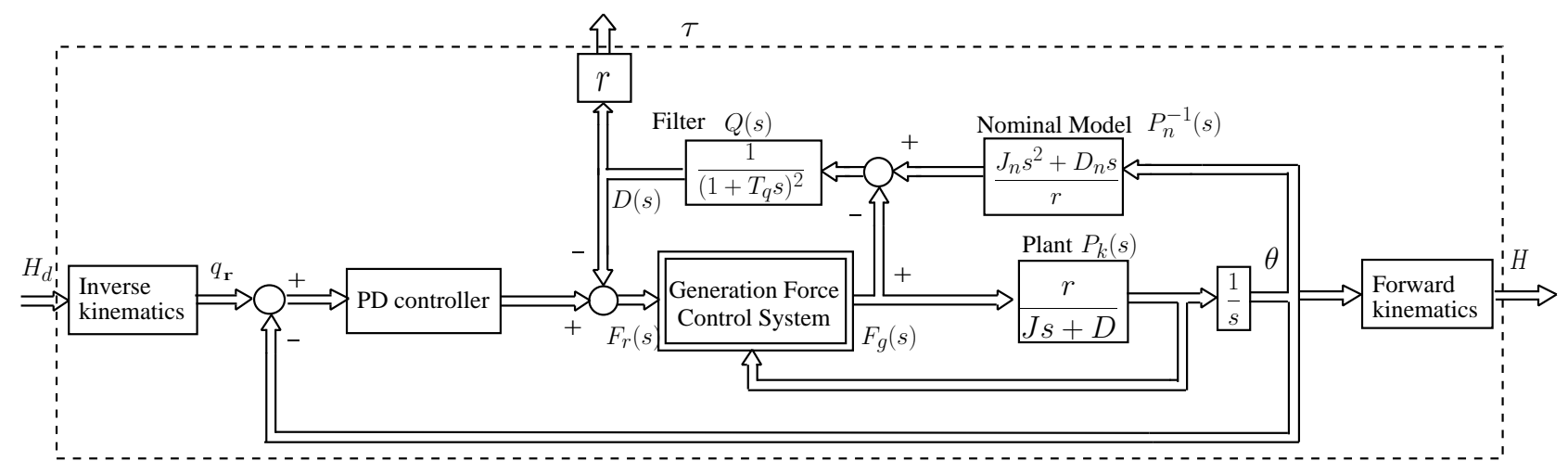

(b) Joint servo system

Fig. 5 Proposed control system

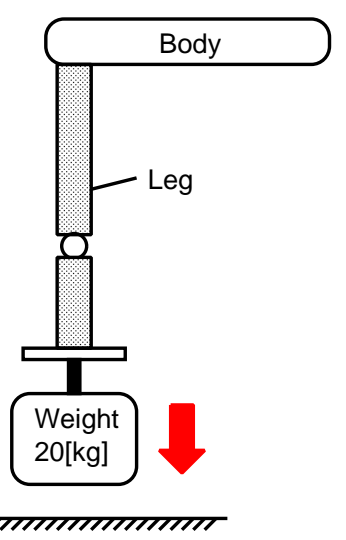

(a) experimental setup

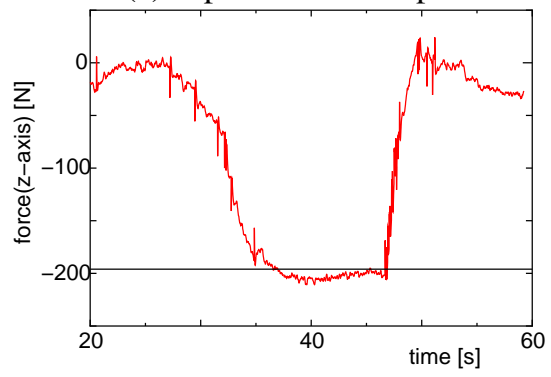

(b) estimated force

Fig. 7 Force estimation property

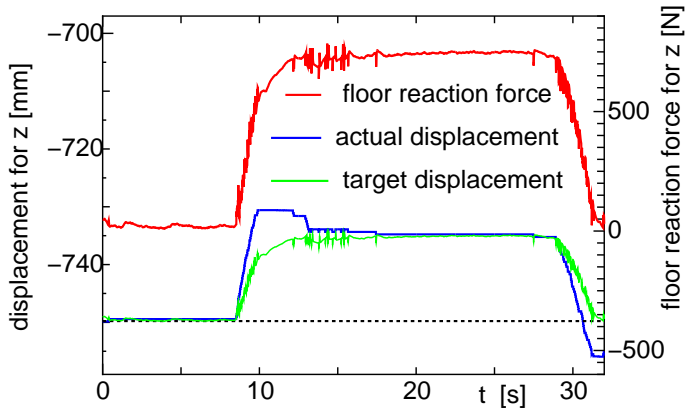

Fig. 8 Compliance control property

tip of a leg and is removed. Figure (b) shows the element for $z$ direction of the estimated floor reaction force. Almost satisfactory estimating performance can be seen.

Fig. 8 shows a compliance control property of a forward right side leg. From the initial state suspended by a crane, the robot is slowly lowered on a floor and stands with 4 legs for a while and then lifted up. A red line shows a floor reaction force normal for the floor plane. Green line indicates a reference displacement for $z$ direction of the leg tip and blue one shows an actual $z$ position of leg tip. In a steady state, the blue line agrees with green one, which shows the given compliance is well realized.

4.2 Equalization of floor reaction force In order to realize a stable standing on a ground, floor reaction force should have the appropriate value. Namely if the center of 


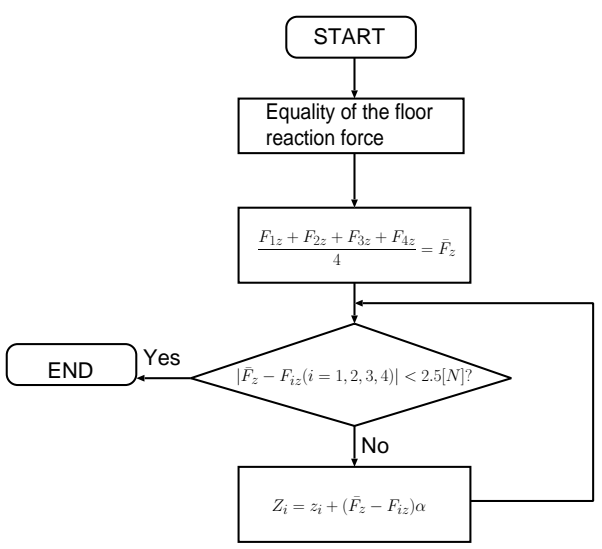

Fig. 9 Flow chart

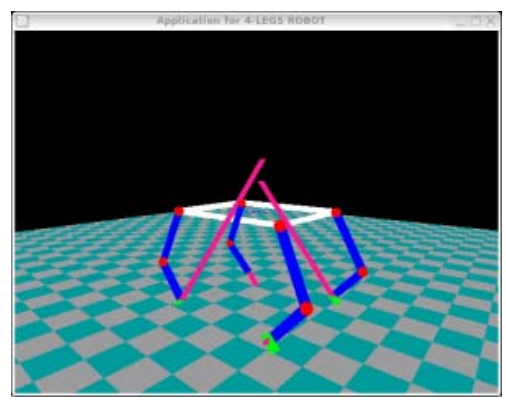

(a)

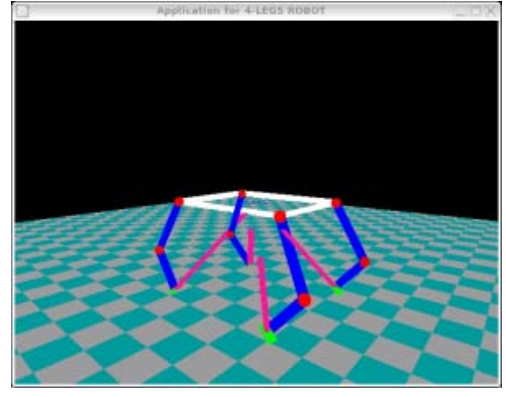

(b)

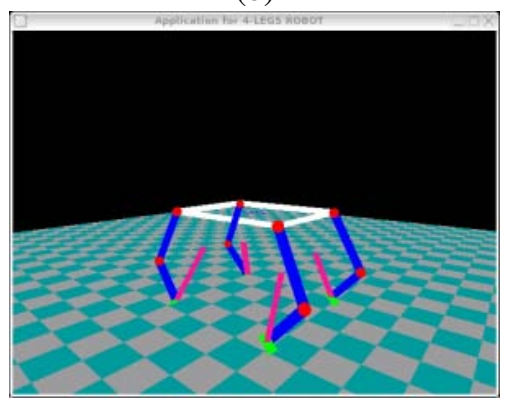

(c)

Fig. 10 Equalization of floor reaction force

gravity of the robot places at the center point of the boundary made by the leg tips, the $z$ element of floor reaction force should be the same. Fig.9 shows a flow chart of equalization. After calculating an average of the $z$ element of floor reaction force and tip of the leg is regulated according to the deviation of a floor reaction force from the average.

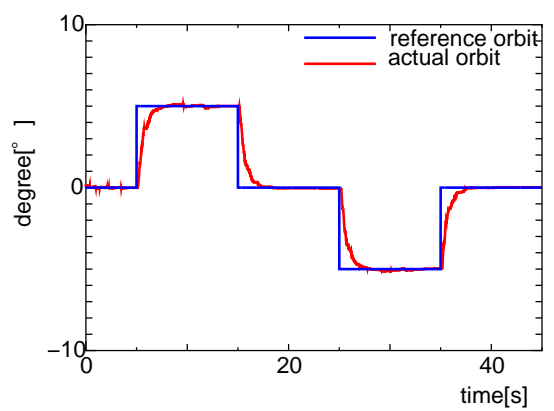

(a) $\operatorname{pitch}(\theta)$ direction

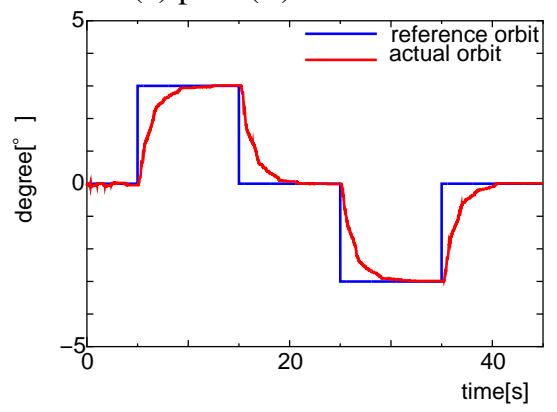

(b) $\operatorname{yaw}(\psi)$ direction

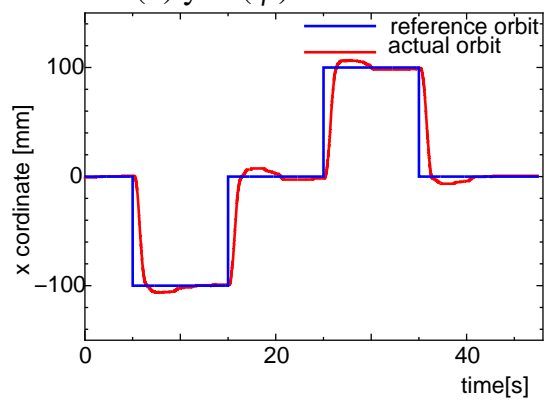

(c) $x$ direction

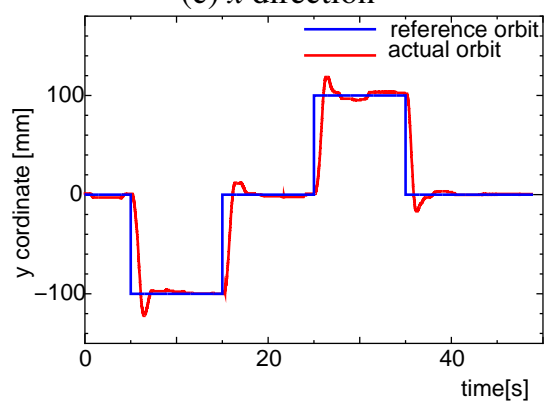

(d) $y$ direction

Fig. 11 Step response

Fig.10 shows the scene of the equalization of floor reaction force by using a computer graphics, where red lines indicate floor reaction force vector. The actual motion of the leg and the actual floor reaction force are reflected in real-time on the computer graphics. We can see the aspect that the norm of reaction force vector converges to their average.

4.3 Position and orientation control Fig.11 shows step responses for each direction. Owing to the detection and compensation scheme of the position and orientation of a robot shown in Fig.6, tracking error almost can not be 


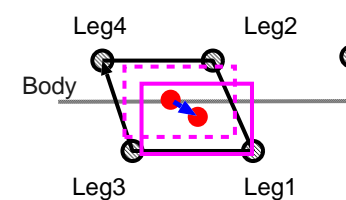

(a)

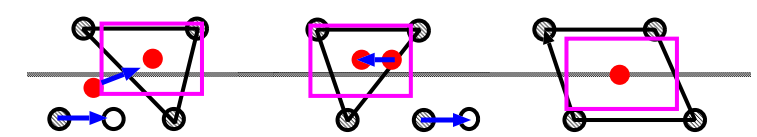

(d)

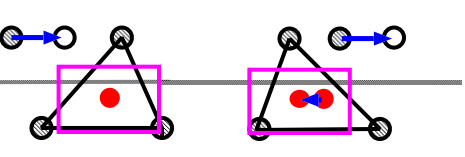

(b)

(e)

(f)
Fig. 12 Intermittent crawl gait

seen in the steady state. In the current strategy, the detected orientation of the body is just a relative one for the ground. Therefore in order to cope with the slope, we are planning to introduce an orientation sensor on the body plane to detect an absolute orientation and, in this case, the proposed detection scheme is utilized to estimate the slope of the ground.

4.4 Static gait We employ the intermediate crawl gait ${ }^{(10)},{ }^{(1)}$ which is the most stable static gait as shown in Fig.12. This walking style has a feature that swing phase and motion phase for changing of center of gravity is completely divided. In the situation (a), we make a body move so that the center of gravity stay in the triangle composed with the supporting legs and then move the swing leg to the proper position. After then we continue the similar motion as (c),(d) and (e). The gait motion of the robot is confirmed but the quantitative results are not shown here.

\section{CONCLUSION}

In this study, we developed a quadruped robot with pneumatic actuators with assumption to apply for the human cooperative tasks.

A compliance control system is composed on the leg without using force/moment sensor but with estimating the floor reaction force by introducing a disturbance observer. If the servo system of an actuator has enough stiffness, then the position control of legs will be able to attain the static gait. But making robot hold compliance for the purpose of above mentioned application, the dynamics change must be compensated. The disturbance observer is also expected as dynamics compensator.

The improvement of gait motion through the enhancement of control performance of leg motion is under the current investigation.

\section{References}

(1) H.Montes,S.Nabulsi,M.Armada,'Detecting ZeroMoment Point in Legged Robot", Proc. of the 7th Int. Conf. CLAWAR 2004

(2) H.Kimura,Y.Fukuoka,A.H.Cohen,'Biologically inspired adaptive walking of a quadruped robot", Philosophical Transactions of The Royal Society A, 365,pp.153170,2007
(3) S.Salmi, A.Halme, "Implementating and Testing a Reasoning-based Free Gait Algorithm in the Six-legged walking Machine "'MECANT", Control Eng. Practice, vol.40,no.4,pp.487-492,1996

(4) J.Estremera,J.A.Cobano,P.Gonzalez de Santos,"Continuous free-crab gaits for hexapod robots on a natural terrain with forbidden zones:An application to humanitarian demining", Robotics and Autonomous System,58,pp.700-711,2010

(5) Ryuichi Hodoshima,Takahiro Doi,Yasushi Fukuda,Shigeo Hirose,Toshihito Okamoto,Junichi Mori,'Development of a Quadruped Walking Robot TITTAN XI for Steep Slope Operation", it J. of Robotics and Mechatronics,vol.19,no.1,2007

(6) John E.Bares, David S.Wettergreen,'Dante II:Technical Description, Results, and Lessons Learned", The Int. J. of Robotics Research,vol.18,no.7,pp.621-649,1999

(7) Stephan K.Chalup,Craig L.Murch,Michael J.Quinlan,"Machine Learning with AIBO Robots in the Four-Legged League of RoboCup", IEEE Trans. on System,Man, and Cybernetics,part C,vol.37,no.3,pp.297310,2007

(8) Shigeo Hirose, Akihiko nagakubo,Ryosei Toyama,"Machine That Can Walk and Climb on Floors, Walls and Ceilings", prroc. of Int. Conf. Advanced Robotics,vol.1,pp.753-758,1991

(9) K.Ohnishi,"Robust Motion Control by Disturbance Observer", Journal of the Robotics Society of Japan,vol.11,no.4,pp.486-493,1993,(in Japanese)

(10) Heeseon Hwang, Youngil youm,"Steady Crawl Gait Generation Algorithm for Quadruped Robots", Advanced Robotics, vol.22,pp.1539-1558,2008

(11) Hideyuki Tsukagoshi, Shigeo Hirose,"Proposal of the Intermittent Crawl Gait and Its Generation", it Journal of the Robotics Society of Japan,vol.17,no.2,pp.301309,1999, (in Japanese) 